\title{
Interior-stress fields produced by a general axisymmetric punch
}

\author{
Longxiang YANG ${ }^{1}$, Zhanjiang WANG ${ }^{2}$, Weiji LIU ${ }^{3}$, Guocheng ZHANG ${ }^{4}$, Bei PENG ${ }^{4,5,}$ \\ ${ }^{I}$ School of Automotive Engineering, Geely University of China, Chengdu 641423, China \\ ${ }^{2}$ Department of Mechanical Engineering, Southwest Jiaotong University, Chengdu 610031, China \\ ${ }^{3}$ School of Mechatronic Engineering, Southwest Petroleum University, Chengdu 610500, China \\ ${ }^{4}$ School of Mechanical and Electrical Engineering, University of Electronic Science and Technology of China, Chengdu 611731, China \\ ${ }^{5}$ Center for Robotics, University of Electronic Science and Technology of China, Chengdu 611731, China
}

Received: 27 November 2019 / Revised: 28 December 2019 / Accepted: 23 November 2020

(C) The author(s) 2020 .

\begin{abstract}
This work is a supplement to the work of Sneddon on axisymmetric Boussinesq problem in 1965 in which the distributions of interior-stress fields are derived here for a punch with general profile. A novel set of mathematical procedures is introduced to process the basic elastic solutions (obtained by the method of Hankel transform, which was pioneered by Sneddon) and the solution of the dual integral equations. These processes then enable us to not only derive the general relationship of indentation depth $D$ and total load $P$ that acts on the punch but also explicitly obtain the general analytical expressions of the stress fields beneath the surface of an isotropic elastic half-space. The usually known cases of punch profiles are reconsidered according to the general formulas derived in this study, and the deduced results are verified by comparing them with the classical results. Finally, these general formulas are also applied to evaluate the von Mises stresses for several new punch profiles.
\end{abstract}

Keywords: axisymmetric; Boussinesq problem; interior stress; Hankel transform; dual integral equations

\section{Introduction}

Sneddon [1] obtained a seminal and compact solution of the displacement-load relationship for the axisymmetric Boussinesq problem [2-5] involving a punch with an arbitrary profile. However, he did not present the interior-stress fields. As one special subclass of contact mechanics [6-9], this subject is characterized as an isotropic half-space that is vertically indented by a rigid punch (frictionless), and the surface-stress distribution and contact area are unknown.

In 1904, Huber [10, 11] first derived the full stress fields in the case of a spherical punch that was approximated using a paraboloid with revolution $z=\frac{1}{2} r^{2}$, where the axis of revolution coincided with the $z$-axis. Later, Love employed the potential theory to solve cases of flat-ended [12] and conical [13] indenter. All these three special cases of punch profiles were reconsidered by Harding et al. [14-16], who used the more concise methods of the Hankel transform and the theory of dual integral equations. Following the establishments and availability of fundamental solutions for the governing equations of elasticity [17-21] and the development of diverse mathematical methods [22-27], the more complicated cases of punch profiles $[28,29]$ are studied. In particular, the form of axisymmetric punch $z=A r^{2 n}(n=0,1,2,3, \ldots)$ was separately considered by Shtaerman (the English language version of the results were edited in Gladwell's book [23]) and Deich [30], and the form $z=\sum_{n=0}^{\infty} A r^{2 n}$ was studied by Deresiewicz [31]. The case of $z=A r^{n}$ $(n=0,1,2,3, \ldots)$ was addressed by Lure and

* Corresponding author: Bei PENG, E-mail: beipeng@uestc.edu.cn 


\begin{tabular}{|c|c|c|c|}
\hline \multicolumn{4}{|c|}{ Nomenclature } \\
\hline$a$ & Contact radius & $R$ & Radius of a spherical indenter \\
\hline$A$ & Shape parameter of the indenter & $u_{r}, u_{z}$ & Elastic displacement in two directions \\
\hline$D$ & Indentation depth & $z, r, \theta$ & Cylindrical coordinates in the spatial domai \\
\hline$E, v$ & Young's modulus and Poisson ratio & $z(r)$ & Profile function of the indenter \\
\hline$g(x)$ & $\begin{array}{l}\text { Displacement function of the surface of a } \\
\text { half-space }\end{array}$ & $\lambda, \mu$ & $\begin{array}{l}\text { Lame constant } \lambda=E v /[(1+v)(1-2 v)] \\
\mu=E /[2(1+v)]\end{array}$ \\
\hline$H_{n}[f(x)]$ & $\begin{array}{l}\text { Hankel transform of order } n \text { of function } \\
f(x)\end{array}$ & $\zeta$ & $\begin{array}{l}\text { Hankel-transformed variable with respect } \\
\text { to } r\end{array}$ \\
\hline \multirow{3}{*}{$\begin{array}{l}J_{n}(x) \\
k\end{array}$} & Bessel function of the first kind with order $n$ & $\psi(\zeta)$ & Undetermined function involving the effect \\
\hline & Shape parameter of the indenter that indicates & & of load and profile of the punch \\
\hline & the times of the power function & $\rho$ & Normalized parameter used in relationship \\
\hline \multirow[t]{3}{*}{$m, n$} & Shape parameters of the indenter that indicates & & $r=\rho a$ \\
\hline & the times of the power function in the form & $\beta(x)$ & Beta function \\
\hline & of $m / n$ & $\Gamma(x)$ & Gamma function \\
\hline$P$ & Total load acting on the punch & \multicolumn{2}{|c|}{$\sigma_{r r}, \sigma_{\theta \theta}, \tau_{z r}, \sigma_{z z} \quad$ Four elastic-stress components } \\
\hline$p_{0}$ & Peak Hertzian contact pressure & $\alpha$ & Inclination angle of the conical indenter \\
\hline
\end{tabular}

Shtaerman (the English language version of the results were edited in Gladwell's book [23]), where the relationships of $P-a$ (contact radius) $-D$ and surface stress were obtained. The case of $z=\sum_{n=0}^{\infty} A r^{n}$ was also investigated by Galin (the English language version of the results were edited in Gladwell's book [23]). Furthermore, the case of $z=A r^{n}(n=1.5,2.5,3.5, \ldots)$ was considered by Woirgard et al. [32]. Significantly, the milestone work for the arbitrary profile of a punch was accomplished by Sneddon [1] in 1965. Additionally, without solving the field equations of elasticity but by employing the methods of cumulative superposition and conservation integrals, Hill and Storakers [33] obtained the results similar to those by Sneddon [1]. Furthermore, the equivalent results were obtained using the method of dimensionality reduction used by Geike [34] and Popov [35]. Recently, the Hertzian contact theory and the superposition-principle method have been employed by Willert et al. [36], who presented the stress characteristics in a contact plane.

Regrettably, except for the known expressions of load $P$, indentation depth $D$, and normal surface stress obtained from the aforementioned studies, the explicit and concise formulas of interior-stress fields have not been integrally derived. These subjects may either have not been pursued, only a speculative sketch was presented, the implicit expressions were given, the mathematical procedures were quite complicated, or simply a few special cases of punch profiles were considered.

In the present study, the relationships of $D, a$, and $P$ and the full components of the interior stresses are derived for the more general profile of a punch, i.e.,

$$
z=A r^{m / n}(m=0,1,2,3, \ldots ; n=1,2,3, \ldots)
$$

which can be reduced to not only the known cases $(m / n=0,1,1.5,2,2.5,3.5,4,4.5, \ldots)$ but also some particular cases such as $4 / 3,5 / 6$, and $2 / 3$ that cannot be dealt using parameter $n$ only. The consideration of this form also comes from some engineering applications, e.g., the minimum dumping profile (4/3) of a carrier as it moves in a fluid medium.

Methodologically, the basic solutions by Sneddon [21], the theory of dual integral equations [37], and the boundary conditions adopted in the present work are the same as those used by Sneddon [1] except for the whole mathematical procedures. Sneddon [1] 
applied a critical relationship of the property on the Hankel transform of, i.e., $\frac{1}{x} \frac{\mathrm{d}}{\mathrm{d} x}\left\{x H_{1}\left[\zeta^{-1} g(\zeta) ; x\right]\right\}=$ $H_{0}[g(\zeta) ; x]$, to deal with the arbitrary profile of a punch. Instead of utilizing such complicated formula with its cumbersome derivations, a novel set of simpler and more ingenious mathematical methods are employed in the present study for this special issue, which enable us to directly obtain results similar to those by Sneddon [1] and to further fully obtain the interior-stress fields.

The general expressions of indentation depth $D$, normal surface stress, total load $P$, and all the interiorstress components are derived for the punch profile of $z=A r^{m / n}$ in this study. In particular, the common cases of $m=0(m / n=0)$ (flat-ended), $m / n=1$ (conical), $m / n=2$ (spherical), and $m / n=k$ of the punch profiles are re-deduced in the current derivations. Finally, the von Mises stresses for the cases of punch profiles $m / n=$ $1,4 / 3,2,4$ are computed according to the explicit and analytical expressions of the interior-stress components in the present work.

\section{General solutions of the axisymmetric- Boussinesq problems}

\subsection{Basic solution}

According to the general solutions of the equilibrium equations of elasticity in cylindrical coordinates, which were solved and summarized by Sneddon [21], the expressions of the interior-stress fields indented by a given axisymmetric profile of a punch can be logically obtained. To more easily solve the current problem, these solutions are modified into the form $\psi(\zeta)$ (the detailed derivations are presented in the Appendix). Then, the non-zero components of the displacements and stresses can be expressed as follows:

$$
\begin{aligned}
& u_{z}(r, z)=H_{0}\left[\psi(\zeta)\left(\frac{\lambda+\mu}{\lambda+2 \mu} \frac{z}{a}+\frac{1}{\zeta}\right) \zeta^{-1} \mathrm{e}^{-\frac{z \zeta}{a}} ; \zeta \rightarrow r\right] \\
& u_{r}(r, z) \\
& =H_{1}\left[\psi(\zeta)\left(\frac{\lambda+\mu}{\lambda+2 \mu} \frac{\zeta}{a} z-\frac{\mu}{\lambda+2 \mu}\right) \zeta^{-2} \mathrm{e}^{-\frac{\zeta}{a} z} ; \zeta \rightarrow r\right]
\end{aligned}
$$

$$
\begin{aligned}
& \sigma_{z z}(r, z) \\
& =-\frac{2 \mu(\lambda+\mu)}{\lambda+2 \mu} H_{0}\left[\psi(\zeta)\left(1+\frac{\zeta}{a} z\right) \frac{1}{\zeta a} \mathrm{e}^{-\frac{\zeta}{a} z} ; \zeta \rightarrow r\right] \\
& \sigma_{\theta \theta}(r, z) \\
& =-\frac{2 \lambda \mu}{\lambda+2 \mu} H_{0}\left[\psi(\zeta) \frac{1}{\zeta a} \mathrm{e}^{-\frac{\zeta}{a} z} ; \zeta \rightarrow r\right] \\
& -\frac{2 \mu(\lambda+\mu)}{r(\lambda+2 \mu)} H_{1}\left[\psi(\zeta) \zeta^{-2}\left(\frac{\mu}{\lambda+\mu}-\frac{\zeta}{a} z\right) \mathrm{e}^{-\frac{\zeta}{a} z} ; \zeta \rightarrow r\right]
\end{aligned}
$$

$$
\begin{aligned}
& \sigma_{r r}(r, z) \\
& =-\frac{2 \mu(\lambda+\mu)}{\lambda+2 \mu} H_{0}\left[\psi(\zeta)\left(1-\frac{\zeta}{a} z\right) \frac{1}{a \zeta} \mathrm{e}^{-\frac{\zeta}{a} z} ; \zeta \rightarrow r\right] \\
& +\frac{2 \mu(\lambda+\mu)}{r(\lambda+2 \mu)} H_{1}\left[\psi(\zeta)\left(\frac{\mu}{\lambda+\mu}-\frac{\zeta}{a} z\right) \zeta^{-2} \mathrm{e}^{-\frac{\zeta}{a} z} ; \zeta \rightarrow r\right] \\
& \tau_{z r}(r, z)=\frac{-2 \mu(\lambda+\mu)}{\lambda+2 \mu} H_{1}\left[\psi(\zeta) \frac{z}{a^{2}} \mathrm{e}^{-\frac{\zeta}{a}} ; \zeta \rightarrow r\right]
\end{aligned}
$$

where $\psi(\zeta)$ denotes the function that reflects the effect of the load and punch profile (as expressed in Eq. (A6) in the Appendix). $\lambda$ and $\mu$ are the Lame constants, and $a$ is the radius of the contact area. Further,

$$
H_{n}[f(\zeta, z) ; \zeta \rightarrow r]=\int_{0}^{\infty} \zeta f(\zeta, z) J_{n}\left(\frac{\zeta}{a} r\right) \mathrm{d} \zeta
$$

where operator $H_{n}[\bullet]$ represents the Hankel transform of order $n$ of function $f(\zeta, z)$, and $J_{n}(\bullet)$ is the Bessel function of the first kind of order $n$.

\subsection{Indentation depth $D(a)$, normal surface stress, and total load $P$}

By combining the aforementioned solutions (as expressed in Eqs. (2)-(7)) with certain necessary boundary conditions, the related axisymmetric Boussinesq problem can be accordingly re-solved. The boundary conditions investigated in the present study can be expressed [1] as follows:

$$
\begin{gathered}
u_{z}(0<r<a, z=0)=D-z(r) \\
\sigma_{z z}(r>a, z=0)=0
\end{gathered}
$$

where $D$ denotes the indentation depth and $z(r)$ is a 
function of the punch profile.

By substituting Eq. (1) and the boundary conditions of Eqs. (9) and (10) into Eqs. (2) and (4) respectively, the following equations can be obtained:

$$
u_{z}(r, z=0)=\int_{0}^{\infty} \psi(\zeta) \zeta^{-1} J_{0}\left(\frac{\zeta r}{a}\right) \mathrm{d} \zeta=D-A r^{m / n}, 0<r<a
$$

$\sigma_{z z}(r, z=0)=\frac{-2 \mu(\lambda+\mu)}{a(\lambda+2 \mu)} \int_{0}^{\infty} \psi(\zeta) J_{0}\left(\frac{\zeta r}{a}\right) \mathrm{d} \zeta=0, \quad r>a$

If the normalized relationship of $r=\rho a$ is considered, Eqs. (11) and (12) can be reduced to the well-known dual integral equations as follows:

$$
\left.\begin{array}{cc}
\int_{0}^{\infty} \zeta^{-1} \psi(\zeta) J_{0}(\zeta \rho) \mathrm{d} \zeta=D-A(a \rho)^{m / n}, & 0<\rho<1 \\
\int_{0}^{\infty} \psi(\zeta) J_{0}(\zeta \rho) \mathrm{d} \zeta=0, & \rho>1
\end{array}\right\}
$$

The solution of Eq. (13) was obtained by Sneddon [37]:

$$
\begin{aligned}
& \psi(\zeta) \\
& =\frac{2 \zeta}{\pi}\left[\cos \zeta \int_{0}^{1} \frac{\rho g(\rho) \mathrm{d} \rho}{\sqrt{1-\rho^{2}}}+\zeta \int_{0}^{1} \frac{u \mathrm{~d} u}{\sqrt{1-u^{2}}} \int_{0}^{1} t g(t u) \sin (\zeta t) \mathrm{d} t\right]
\end{aligned}
$$

where functions of $g(\rho)$ and $g(t u)$ in the condition are expressed as

$$
\begin{aligned}
& g(\rho)=D-A(a \rho)^{m / n} \\
& g(t u)=D-A(a t u)^{m / n}
\end{aligned}
$$

By substituting Eqs. (15) and (16) into Eq. (14), function $\psi(\zeta)$ can be pre-digested as

$$
\begin{aligned}
& \psi(\zeta)=\frac{A}{\pi} \\
& \times\left\{\frac{2 D}{A} \sin \zeta-a^{\frac{m}{n}} \beta\left(\frac{2 n+m}{2 n}, \frac{1}{2}\right)\left[\zeta \cos \zeta+\zeta^{2} \int_{0}^{1} t^{\frac{m+n}{n}} \sin (\zeta t) \mathrm{d} t\right]\right\}
\end{aligned}
$$

The abovementioned processes represent the classical steps in solving this type of problems by employing the Hankel transform [1, 14-16, 21, 32].
However, no attempt can be found in the known literature in which Eq. (17) is further transformed into a manageable form. In the present study, the existing integral relationship [38] is

$$
\int x^{k} \sin x \mathrm{~d} x=x^{k-1}(k \sin x-x \cos x)-k(k-1) \int x^{k-2} \sin x \mathrm{~d} x
$$

Then, the integral term of Eq. (17) can be transformed into:

$$
\begin{aligned}
& \int_{0}^{1} t^{\frac{m+n}{n}} \sin (\zeta t) \mathrm{d} t \\
& =\frac{1}{\zeta^{2}}\left[\frac{(m+n) \sin \zeta}{n}-\zeta \cos \zeta-\frac{m(m+n)}{n^{2}} \int_{0}^{1} t^{\frac{m-n}{n}} \sin (\zeta t) \mathrm{d} t\right]
\end{aligned}
$$

Therefore, Eq. (17) can be rewritten as

$$
\psi(\zeta)=\left(\frac{2 D}{\pi}-\frac{C_{1} n}{m}\right) \sin \zeta+C_{1} \chi(\zeta)
$$

where

$$
\begin{gathered}
C_{1}=\frac{A}{\pi} \frac{m(m+n)}{n^{2}} \beta\left(\frac{2 n+m}{2 n}, \frac{1}{2}\right) a^{\frac{m}{n}} \\
\chi(\zeta)=\int_{0}^{1} t^{\frac{m-n}{n}} \sin (\zeta t) \mathrm{d} t
\end{gathered}
$$

where $\beta(\cdot)$ denotes the beta function.

The obtainment of Eq. (20) is the key step in this derivation. These processes are completely different from the corresponding procedures employed by Sneddon [1, 14-16, 21] and Woirgard et al. [32], which either greatly blocked the derivation of expressions of the interior stresses [1], limited the general discussions of the punch profiles [14-16, 21], or complicated the expressions of the interior-stress fields [32].

Once the explicit expression of function $\psi(\zeta)$ has been obtained, all the components of the displacements and stresses in Eqs. (2)-(7) can be subsequently computed. However, substituting Eq. (20) into the basic solutions of Eqs. (2)-(7) would lead to extremely complicated forms of integral equation, which make them even impossible to calculate. In fact, the form $\psi(\zeta)$ can be simplified by considering the circumstance of a normal surface stress on the contact periphery. Substituting Eq. (20) into Eq. (12) yields: 


$$
\begin{aligned}
& \sigma_{z z}(r, z=0) \\
& =\frac{-2 \mu(\lambda+\mu)}{a(\lambda+2 \mu)} \int_{0}^{\infty}\left[\left(\frac{2 D}{\pi}-\frac{C_{1} n}{m}\right) \sin \zeta+C_{1} \chi(\zeta)\right] J_{0}\left(\frac{\zeta r}{a}\right) \mathrm{d} \zeta
\end{aligned}
$$

Simultaneously, two types of axisymmetric punch profiles should be respectively addressed: (a) $m \neq 0$ $(m / n \neq 0)$ and (b) $m=0(m / n=0)$. Furthermore, as we can observe in the following, the inter-relationships of indentation depth $D$, normal surface stress $\sigma_{z z}(0<$ $r \leq a, z=0)$, and total load $P$ can be derived as the obtainment of simplified expression $\psi(\zeta)$.

\section{(a) $m \neq 0(m / n \neq 0)$}

Obviously, the case of $m \neq 0(m / n \neq 0)$ corresponds to the incomplete contact problem, which means that the normal surface stress on the periphery of the contact region is assumed to be zero, i.e., $\sigma_{z z}(r=a, z=0)=0$, when adhesion is not considered. Therefore, by setting $r=a$ in Eq. (23), we can obtain:

$$
\begin{aligned}
& \sigma_{z z}(r=a, z=0) \\
& =\frac{-2 \mu(\lambda+\mu)}{(\lambda+2 \mu) a}\left[\begin{array}{l}
C_{1} \int_{0}^{1} t^{\frac{m-n}{n}} \mathrm{~d} t \int_{0}^{\infty} \sin (\zeta t) J_{0}(\zeta) \mathrm{d} \zeta \\
+\left(\frac{2 D}{\pi}-\frac{C_{1} n}{m}\right) \int_{0}^{\infty} \sin \zeta J_{0}(\zeta) \mathrm{d} \zeta
\end{array}\right]=0
\end{aligned}
$$

We note that the known results of the integral equations [38] are

$$
\begin{gathered}
\int_{0}^{\infty} \sin (\zeta t) J_{0}(\zeta) \mathrm{d} \zeta=0, \quad 0<t<1 \\
\int_{0}^{\infty} \sin \zeta J_{0}(\zeta) \mathrm{d} \zeta=\infty
\end{gathered}
$$

Thus, we obtain the following limitation:

$$
\frac{2 D}{\pi}-\frac{C_{1} n}{m}=0
$$

Therefore, the indentation depth produced by axisymmetric punch profile $z=A r^{m / n}(m=n=1,2$, $3, . .$.$) can be obtained as$

$$
D=\frac{A}{2} \frac{m+n}{n} \beta\left(\frac{2 n+m}{2 n}, \frac{1}{2}\right) a^{\frac{m}{n}}
$$

Moreover, by introducing Eq. (27) back into Eq. (23), the normal surface stress can be reduced and calculated as

$$
\sigma_{z z}(r, z=0)=\frac{-E}{1-v^{2}} \frac{C_{1}}{2 a} \int_{\frac{r}{a}}^{1} \frac{t^{\frac{m-n}{n}}}{\sqrt{t^{2}-\frac{r^{2}}{a^{2}}}} \mathrm{~d} t, \quad 0<r<a
$$

where Lame constants $\lambda$ and $\mu$ are replaced by Young modulus $E$ and Poisson's ratio $v$ according to the following relationships [11]:

$$
\lambda=\frac{E v}{(1+v)(1-2 v)}, \quad \mu=\frac{E}{2(1+v)}
$$

Then, total load $P$ can be obtained from Eq. (29), i.e.,

$$
P=-2 \pi \int_{0}^{a} \sigma_{z z}(r, z=0) r \mathrm{~d} r=\frac{E}{1-v^{2}} \frac{2 a m}{m+n} D, \quad 0<r<a
$$

Additionally, if Eq. (28) is substituted into Eq. (31), the dependence of applied load $P$ on contact radius $a$ only can be expressed as

$$
P=\frac{E}{1-v^{2}} \frac{A m}{n} \beta\left(\frac{2 n+m}{2 n}, \frac{1}{2}\right) a^{\frac{m+n}{n}}
$$

Finally, function $\psi(\zeta)$ in Eq. (20) can be simplified using Eq. (27)

$$
\psi(\zeta)=C_{1} \chi(\zeta)
$$

\section{(b) $m=0(m / n=0)$}

This case actually illustrates that the flat-ended punch and the corresponding normal surface stress on the periphery of the contact region can be obtained by setting $m=0$ and $r=a$ in Eq. (23):

$$
\sigma_{z z}^{\text {flat-ended }}(r=a, z=0)=\frac{-4 \mu(\lambda+\mu)}{\lambda+2 \mu} \frac{D}{\pi a} \int_{0}^{\infty} \sin \zeta J_{0}(\zeta) \mathrm{d} \zeta
$$

From Eq. (26), we have

$$
\sigma_{z z}^{\text {flat-ended }}(r=a, z=0)=\infty
$$

This mathematical result reflects the physical phenomenon that indicates that the pressure is infinite at the edge of the complete contact area. The pressure within the contact area can be obtained by substituting 
$m=0$ into Eq. (23):

$$
\sigma_{z z}^{\text {flat-ended }}(r, z=0)=\frac{-E}{1-v^{2}} \frac{D}{\pi} \frac{1}{\sqrt{a^{2}-r^{2}}}, \quad 0<r<a
$$

Then, the total load can be expressed as

$$
\begin{aligned}
P^{\text {flat-ended }} & =-2 \pi \int_{0}^{a} \sigma_{z z}^{\text {flat-ended }}(r, z=0) r \mathrm{~d} r \\
& =\frac{2 E D a}{1-v^{2}}, \quad 0<r<a
\end{aligned}
$$

The results of Eqs. (36) and (37) are identical with the known expressions provided by Sneddon [15].

Finally, the expression for function $\psi(\zeta)$ in Eq. (20) for a flat-ended punch $(A=0, m=0)$ can be calculated as

$$
\psi^{\text {flat-ended }}(\zeta)=\frac{2 D \sin \zeta}{\pi}
$$

\subsection{Interior-stress field}

From simplified function $\psi(\zeta)$ expressed in Eq. (33) and from Eq. (38), the interior-stress fields indented by the punch profile $z=A r^{m / n}$ can be easily calculated from basic solutions of Eqs. (2)-(7). The special case of $m=0(m / n=0)$ will be discussed later (in Section 3). Here, the general stress expressions in the case where $m \neq 0(m / n \neq 0)$ are investigated, which can be integrated by substituting Eq. (33) into Eqs. (4-7):

$$
\begin{gathered}
\sigma_{\theta \theta}(r, z)=\frac{-E}{1-v^{2}} \frac{C_{1}}{2 a} \int_{0}^{1}\left[2 v I_{1}^{0}+\frac{a(1-2 v)}{r} I_{0}^{1}-\frac{z}{r} I_{1}^{1}\right] t^{\frac{m-n}{n}} \mathrm{~d} t \\
\sigma_{r r}(r, z)=\frac{-E}{1-v^{2}} \frac{C_{1}}{2 a} \int_{0}^{1}\left[I_{1}^{0}-\frac{z}{a} I_{2}^{0}+\frac{a(2 v-1)}{r} I_{0}^{1}+\frac{z}{r} I_{1}^{1}\right] t^{\frac{m-n}{n}} \mathrm{~d} t \\
\tau_{z r}(r, z)=\frac{-E}{1-v^{2}} \frac{C_{1}}{2 a} \frac{z}{a} \int_{0}^{1} I_{2}^{1} t^{\frac{m-n}{n}} \mathrm{~d} t \\
\sigma_{z z}(r, z)=\frac{-E}{1-v^{2}} \frac{C_{1}}{2 a} \int_{0}^{1}\left(I_{1}^{0}+\frac{z}{a} I_{2}^{0}\right) t^{\frac{m-n}{n}} \mathrm{~d} t
\end{gathered}
$$

where

$$
I_{q}^{p}(r, z, t)=\int_{0}^{\infty} \zeta^{q-1} J_{p}\left(\frac{r}{a} \zeta\right) \exp ^{-\frac{z}{a} \zeta} \sin (\zeta t) \mathrm{d} \zeta
$$

In particular, the expressions on surface $z=0$ for $0<r<a$ are

$$
\begin{aligned}
& \sigma_{\theta \theta}(r, z=0) \\
& =\frac{-E(1-2 v)}{1-v^{2}} \frac{C_{1}}{2 a}\left[\int_{\frac{r}{a}}^{1}\left(\frac{1}{1-2 v}-\frac{a^{2} t^{2}}{r^{2}}\right) \frac{t^{\frac{m-n}{n}}}{\sqrt{t^{2}-\frac{r^{2}}{a^{2}}}} \mathrm{~d} t+\frac{n}{m+n} \frac{a^{2}}{r^{2}}\right]
\end{aligned}
$$

$\sigma_{r r}(r, z=0)$

$$
\begin{gathered}
=\frac{-E(1-2 v)}{1-v^{2}} \frac{C_{1}}{2 a}\left[\int_{\frac{r}{a}}^{1}\left(\frac{2 v}{1-2 v}+\frac{a^{2} t^{2}}{r^{2}}\right) \frac{t^{\frac{m-n}{n}}}{\sqrt{t^{2}-\frac{r^{2}}{a^{2}}}} \mathrm{~d} t-\frac{n}{m+n} \frac{a^{2}}{r^{2}}\right] \\
\sigma_{z z}(r, z=0)=\frac{-E}{1-v^{2}} \frac{C_{1}}{2 a} \int_{\frac{r}{a}}^{1} \frac{t^{\frac{m-n}{n}}}{\sqrt{t^{2}-\frac{r^{2}}{a^{2}}}} \mathrm{~d} t \\
\tau_{z r}(r, z=0)=0
\end{gathered}
$$

Those at the surface for $r>a$ are expressed as

$$
\begin{gathered}
\sigma_{\theta \theta}(r, z=0)=-\sigma_{r r}(r, z=0)=\frac{-E(1-2 v)}{1-v^{2}} \frac{C_{1} a}{2 r^{2}} \frac{n}{m+n} \\
\tau_{z r}(r, z=0)=\sigma_{z z}(r, z=0)=0
\end{gathered}
$$

The stresses on the axial direction $r=0$ are expressed as

$$
\begin{aligned}
& \sigma_{\theta \theta}(r=0, z)=\sigma_{r r}(r=0, z) \\
& =\frac{E}{1-v^{2}} \frac{C_{1} a}{4}\left[2 z^{2} \int_{0}^{1} \frac{t^{\frac{m}{n}}}{\left(z^{2}+a^{2} t^{2}\right)^{2}} \mathrm{~d} t-(1+2 v) \int_{0}^{1} \frac{t^{\frac{m}{n}}}{z^{2}+a^{2} t^{2}} \mathrm{~d} t\right] \\
& \sigma_{z z}(r=0, z)=\frac{-E}{1-v^{2}} \frac{C_{1}}{2} \int_{0}^{1} \frac{t^{\frac{m}{n}}}{z^{2}+a^{2} t^{2}}\left(a+\frac{2 a z^{2}}{z^{2}+a^{2} t^{2}}\right) \mathrm{d} t \\
& \tau_{z r}(r=0, z)=0
\end{aligned}
$$

Finally, the expressions at original point $r=z=0$ for $m>n$ are

$$
\sigma_{\theta \theta}(r=0, z=0)=\sigma_{r r}(r=0, z=0)=\frac{-E(1+2 v)}{1-v^{2}} \frac{C_{1}}{4 a} \frac{n}{m-n}
$$




$$
\begin{gathered}
\sigma_{z z}(r=0, z=0)=\frac{-E}{1-v^{2}} \frac{C_{1}}{2 a} \frac{n}{m-n} \\
\tau_{z r}(r=0, z=0)=0
\end{gathered}
$$

For $m \leq n$,

$$
\begin{gathered}
\sigma_{\theta \theta}(r=0, z=0)=\sigma_{r r}(r=0, z=0)=\sigma_{z z}(r=0, z=0)=\infty \\
\tau_{z r}(r=0, z=0)=0
\end{gathered}
$$

\section{Results of the special profiles of a punch}

The four special cases of punch profile, namely, $m / n=$ $0(m=0), m / n=1, m / n=2$, and $m / n=k$, which have been studied using other mathematical procedures $[10-16,21,23,32]$, are reconsidered according to the abovementioned general formulas, and the re-deduced results are verified by comparison with the classical results. Furthermore, the distributions of the von Mises stresses for some new cases of punch profiles (such as $4 / 3$ ) will be presented.

\subsection{Flat-ended cylindrical punch $m / n=0(m=0)$}

The expressions for the normal surface stress and total load indented by this flat-ended cylindrical punch have been derived and verified in Section 2, and the distributions of the interior-stress fields in an isotropic half-space can be easily obtained by substituting Eq. (38) into Eqs. (2) and (4)-(7). Thus,

$$
\begin{aligned}
u_{z}(r, z)=\frac{2 D}{\pi} \int_{0}^{\infty}\left(\frac{\lambda+\mu}{\lambda+2 \mu} \frac{z}{a}+\frac{1}{\zeta}\right) \sin \zeta \mathrm{e}^{-\frac{z \zeta}{a}} J_{0}\left(\frac{\zeta r}{a}\right) \mathrm{d} \zeta \\
\sigma_{\theta \theta}(r, z)=-\frac{4 \mu}{\lambda+2 \mu} \frac{D}{\pi} \int_{0}^{\infty}\left[\begin{array}{l}
\frac{\lambda}{a} J_{0}\left(\frac{\zeta r}{a}\right) \\
\left.+\frac{\mu}{r}\left(\zeta^{-1}-\frac{\lambda+\mu}{\mu} \frac{z}{a}\right) J_{1}\left(\frac{\zeta r}{a}\right)\right]
\end{array}\right. \\
\times \sin \zeta \mathrm{e}^{-\frac{z \zeta}{a}} \mathrm{~d} \zeta \\
\sigma_{r r}(r, z)=-\frac{4 \mu}{\lambda+2 \mu} \frac{D}{\pi} \int_{0}^{\infty}\left[\begin{array}{l}
\frac{\lambda+\mu}{a}\left(1-\frac{z \zeta}{a}\right) J_{0}\left(\frac{\zeta r}{a}\right) \\
-\frac{\mu}{r}\left(\zeta^{-1}-\frac{\lambda+\mu}{\mu} \frac{z}{a}\right) J_{1}\left(\frac{\zeta r}{a}\right)
\end{array}\right] \\
\times \sin \zeta \mathrm{e}^{-\frac{z \zeta}{a}} \mathrm{~d} \zeta
\end{aligned}
$$

$$
\begin{aligned}
\tau_{z r}(r, z)= & \frac{-4 \mu(\lambda+\mu)}{\lambda+2 \mu} \frac{D z}{\pi a^{2}} \int_{0}^{\infty} \zeta J_{1}\left(\frac{\zeta r}{a}\right) \sin \zeta \mathrm{e}^{-\frac{z \zeta}{a}} \mathrm{~d} \zeta \\
\sigma_{z z}(r, z)= & \frac{-4 \mu(\lambda+\mu)}{\lambda+2 \mu} \frac{D}{\pi a} \int_{0}^{\infty}\left(1+\frac{z \zeta}{a}\right) J_{0}\left(\frac{\zeta r}{a}\right) \\
& \times \sin \zeta \mathrm{e}^{-\frac{z \zeta}{a}} \mathrm{~d} \zeta
\end{aligned}
$$

If the notations adopted in the aforementioned formulas are used to perform the following replacements,

$$
D=\varepsilon ; \zeta=p ; \frac{z}{a}=\zeta ; \frac{r}{a}=\rho
$$

then Eqs. (58)-(62) can be found to be identical to the corresponding expressions provided by Sneddon [21].

\subsection{Conical punch $(m / n=1)$}

For the case of the conical punch shown in Fig. 1, all the stress information involving this punch profile can be computed when its shape parameters ( $A=\cot \alpha$ and $m / n=1)$ are substituted into Eqs. (28), (29), (32), and (39)-(42). The expression of the indentation depth can be obtained as follows:

$$
D=\frac{\pi a \cot \alpha}{2}
$$

The normal surface stress is expressed as

$$
\sigma_{z z}(r, z=0)=\frac{-E}{1-v^{2}} \frac{\cot \alpha}{2} \operatorname{arccosh}\left(\frac{a}{r}\right)
$$

Then, the total load contains:

$$
P=\frac{E}{1-v^{2}} \frac{\pi a^{2} \cot \alpha}{2}
$$

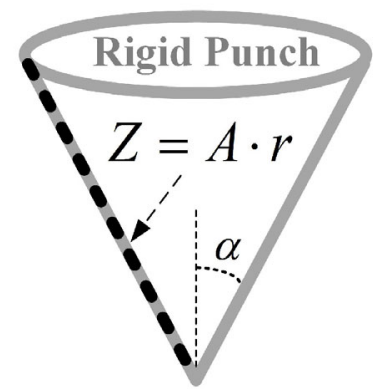

Fig. 1 Conical rigid punch. 
and the components of the interior stresses are expressed as

$$
\begin{aligned}
& \sigma_{\theta \theta}(r, z)=\frac{-E}{1-v^{2}} \frac{\cot \alpha}{2} \int_{0}^{\infty}\left\{\begin{array}{l}
2 v J_{0}\left(\frac{r \zeta}{a}\right) \\
+\left[\frac{a(1-2 v)}{r \zeta}-\frac{z}{r}\right] J_{1}\left(\frac{r \zeta}{a}\right)
\end{array}\right\} \\
& \times \frac{1-\cos \zeta}{\zeta} \mathrm{e}^{-\frac{z \zeta}{a}} \mathrm{~d} \zeta \\
& \begin{aligned}
& \sigma_{r r}(r, z)= \frac{-E}{1-v^{2}} \frac{\cot \alpha}{2} \int_{0}^{\infty}\left\{\begin{array}{l}
\left(1-\frac{z \zeta}{a}\right) J_{0}\left(\frac{r \zeta}{a}\right) \\
+\left[\frac{a(2 v-1)}{r \zeta}+\frac{z}{r}\right] J_{1}\left(\frac{r \zeta}{a}\right)
\end{array}\right\} \\
& \times \frac{1-\cos \zeta}{\zeta} \mathrm{e}^{-\frac{z \zeta}{a}} \mathrm{~d} \zeta \quad(68) \\
& \tau_{z r}(r, z)=\frac{-E}{1-v^{2}} \frac{\cot \alpha}{2} \frac{z}{a} \int_{0}^{\infty}(1-\cos \zeta) J_{1}\left(\frac{r \zeta}{a}\right) \mathrm{e}^{-\frac{z \zeta}{a}} \mathrm{~d} \zeta
\end{aligned} \\
& \begin{array}{l}
\sigma_{z z}(r, z) \\
=\frac{-E}{1-v^{2}} \frac{\cot \alpha}{2} \int_{0}^{\infty}\left(1+\frac{z \zeta}{a}\right) J_{0}\left(\frac{r \zeta}{a}\right) \frac{1-\cos \zeta}{\zeta} \mathrm{e}^{-\frac{z \zeta \zeta}{a}} \mathrm{~d} \zeta
\end{array}
\end{aligned}
$$

The results of Eqs. (64)-(66) are consistent with those of the classical formulas in Refs. [1, 23, 39]. The expressions of the interior stresses in Eqs. (67)-(70) are identical to those given by Sneddon $[16,21]$ if the following notation substitutions are performed:

$$
a \cot \alpha=\varepsilon ; \zeta=p ; \frac{z}{a}=\zeta ; \frac{r}{a}=\rho
$$

We need to note that the functions of the abovementioned two punch profiles (flat-ended and conical) are expanded into a combination of polynomials of $g(\rho)=\sum_{n=0}^{\infty} A_{n} \cdot \rho^{n}$ in Refs. $[15,16,21,32]$, which are completely different from the adopted method in the current study.

\subsection{Approximated spherical punch $(m / n=2)$}

Actually, the original contact problem studied by Hertz [6] was induced by an approximate spherical punch where the shape parameters of this punch profile could be expressed as $z=A r^{m / n}(A=1 / 2 R, m / n=2)$. By combining this expression with Eq. (28), the indentation depth in this case can be obtained.

$$
D=\frac{a^{2}}{R}
$$

The total load can be derived from Eq. (32),

$$
P=\frac{E}{1-v^{2}} \frac{4 a^{3}}{3 R}
$$

The interior-stress components can be calculated by substituting these shape parameters into Eqs. (39)-(57). Thus, we have:

$$
\begin{gathered}
\sigma_{\theta \theta}(r, z)=\frac{-E}{1-v^{2}} \frac{2 a}{\pi R} \int_{0}^{1}\left[2 v I_{1}^{0}+\frac{a(1-2 v)}{r} I_{0}^{1}-\frac{z}{r} I_{1}^{1}\right] t \mathrm{~d} t \\
\sigma_{r r}(r, z) \\
=\frac{-E}{1-v^{2}} \frac{2 a}{\pi R} \int_{0}^{1}\left[I_{1}^{0}+\frac{z}{r} I_{1}^{1}+\frac{a(2 v-1)}{r} I_{0}^{1}-\frac{z}{a} I_{2}^{0}\right] t \mathrm{~d} t \\
\tau_{z r}(r, z)=\frac{-E}{1-v^{2}} \frac{2 a}{\pi R} \frac{z}{a} \int_{0}^{1} I_{2}^{1} t \mathrm{~d} t \\
\sigma_{z z}(r, z)=\frac{-E}{1-v^{2}} \frac{2 a}{\pi R} \int_{0}^{1}\left(I_{1}^{0}+\frac{z}{a} I_{2}^{0}\right) t \mathrm{~d} t
\end{gathered}
$$

where $I_{q}^{p}(r, z, t)$ is expressed similar to that in Eq. (43).

The expressions on surface $z=0$ for $0<r \leq a$ are

$$
\begin{gathered}
\sigma_{\theta \theta}(r, z=0) \\
=\frac{-E}{1-v^{2}} \frac{2 a}{\pi R}\left\{\frac{1-2 v}{3} \frac{a^{2}}{r^{2}}\left[1-\sqrt{1-\frac{r^{2}}{a^{2}}}\left(1-\frac{r^{2}}{a^{2}}\right)\right]+2 v \sqrt{1-\frac{r^{2}}{a^{2}}}\right\} \\
\sigma_{r r}(r, z=0) \\
=\frac{E}{1-v^{2}} \frac{2 a}{\pi R}\left\{\frac{1-2 v}{3} \frac{a^{2}}{r^{2}}\left[1-\sqrt{1-\frac{r^{2}}{a^{2}}}\left(1-\frac{r^{2}}{a^{2}}\right)\right]-\sqrt{1-\frac{r^{2}}{a^{2}}}\right\} \\
\sigma_{z z}(r, z=0)=\frac{-E}{1-v^{2}} \frac{2 a}{\pi R} \sqrt{1-\frac{r^{2}}{a^{2}}} \\
\tau_{z r}(r, z=0)=0
\end{gathered}
$$

On surface $z=0$ for $r>a$, the expressions are 


$$
\begin{gathered}
\sigma_{\theta \theta}(r, z=0)=-\sigma_{r r}(r, z=0)=\frac{-E}{1-v^{2}} \frac{2 a}{\pi R} \frac{1-2 v}{3} \frac{a^{2}}{r^{2}} \\
\tau_{z r}(r, z=0)=\sigma_{z z}(r, z=0)=0
\end{gathered}
$$

On the $r=0$ axis, the expressions are

$$
\begin{gathered}
\sigma_{\theta \theta}(r=0, z) \\
=\sigma_{r r}(r=0, z) \\
=\frac{-E}{1-v^{2}} \frac{2 a}{\pi R}\left\{(1+v)\left[1-\frac{z}{a} \operatorname{acr} \tan \left(\frac{a}{z}\right)\right]-\frac{1}{2\left(1+\frac{z^{2}}{a^{2}}\right)}\right\} \\
\sigma_{z z}(r=0, z)=\frac{-E}{1-v^{2}} \frac{2 a}{\pi R} \frac{a^{2}}{a^{2}+z^{2}} \\
\tau_{z r}(r=0, z)=0
\end{gathered}
$$

At original point $r=z=0$,

$$
\begin{gathered}
\sigma_{r r}(r=0, z=0)=\sigma_{\theta \theta}(r=0, z=0)=\frac{-E}{1-v^{2}} \frac{2 a}{\pi R} \frac{1+2 v}{2} \\
\sigma_{z z}(r=0, z=0)=\frac{-E}{1-v^{2}} \frac{2 a}{\pi R}
\end{gathered}
$$

$$
\tau_{z r}(r=0, z=0)=0
$$

Obviously, the particular components of the stresses on surface $z=0$, axial $r=0$, and original point $z=r=0$ as expressed in Eqs. (78)-(89) agree with the results by Huber [10] (Huber's results also presented by Hills et al. [11]), which assumes that the peak contact pressure is involved as follows:

$$
p_{0}=\frac{E}{1-v^{2}} \frac{2 a}{\pi R}
$$

Unfortunately, we fail to reduce the integral expressions of Eqs. (74)-(77) to the corresponding Huber forms [10] (Huber's results also presented in Hills [11] where $u^{2}$ should be corrected to $u$ ). However, the numerical solutions of Eqs. (74)-(77) are obtained and compared with the values of the Huber analytical expressions, as shown in Fig. 2.

\subsection{Punch in the form of a paraboloid with revolution $m / n=k$}

In the present work, the widely used formulas of the inter-relationships between indentation depth $D$,
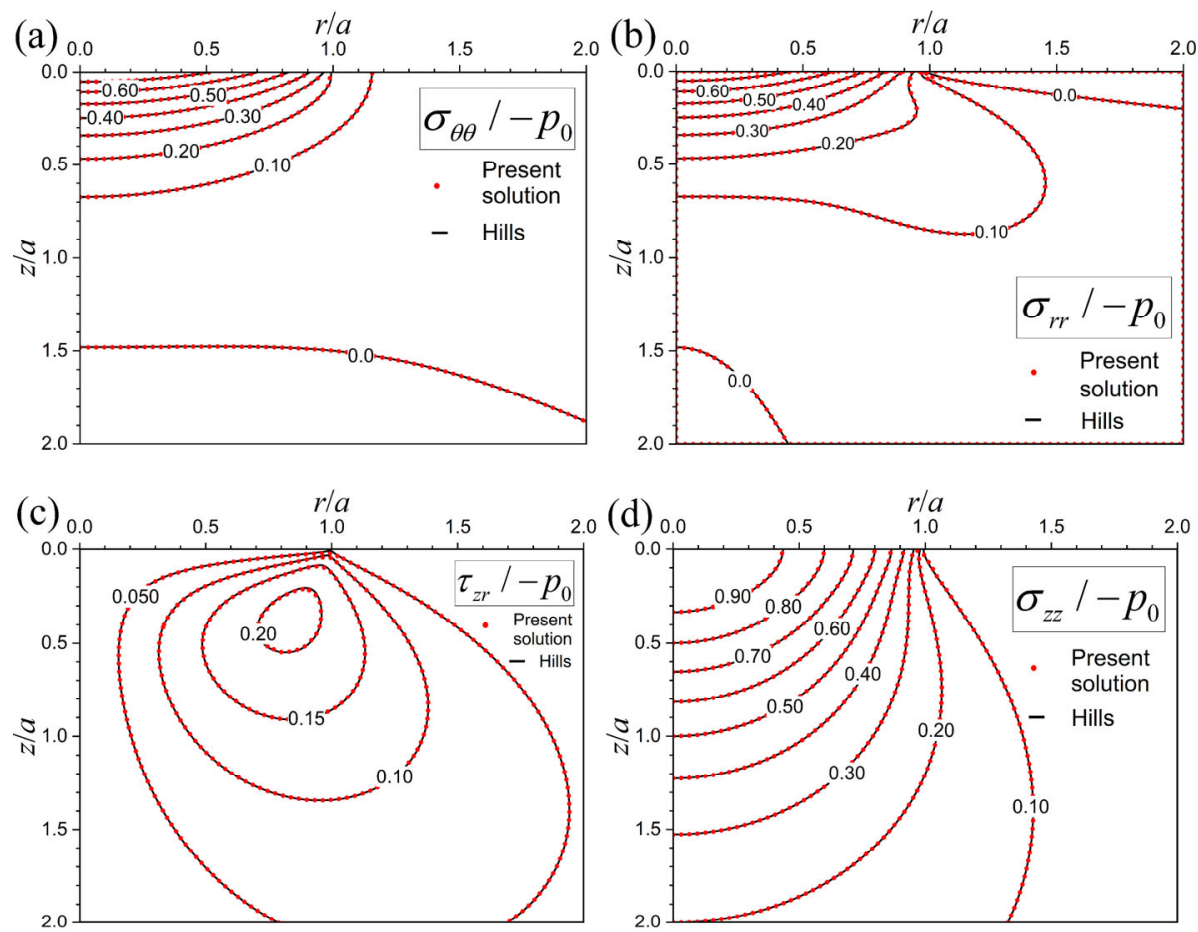

Fig. 2 Numerical comparisons of the interior-stress distributions based on the general formulas in Eqs. (74)-(77) and the classical formulas of Huber [10] (Huber's results are also presented by Hills et al. [11]). For fewer calculation, the common part ( $p_{0}$, as expressed in Eq. (90)) is excluded from these expressions, and some parameters, namely, $R=a=1$ and $v=0.3$, are set. 
contact radius $a$, and total load $P$ are discussed in detail. With the exception of the more general work of Sneddon [1], the classical solutions of Galin (the English language version of the results were edited in Gladwell's book [23]) for monomial punch $z=A r^{k}$ are reduced from the derived formulas here.

According to the relationship between the gamma and beta functions, we have:

$$
\beta\left(\frac{2 n+m}{2 n}, \frac{1}{2}\right)=\frac{m}{m+n} 2^{\frac{m-n}{n}}\left[\Gamma\left(\frac{m}{2 n}\right)\right]^{2}\left[\Gamma\left(\frac{m}{n}\right)\right]^{-1}
$$

The indentation depth is calculated from Eq. (28):

$$
D=\frac{A m}{2 n} 2^{\frac{m-n}{n}}\left[\Gamma\left(\frac{m}{2 n}\right)\right]^{2}\left[\Gamma\left(\frac{m}{n}\right)\right]^{-1} a^{\frac{m}{n}}
$$

By substituting $m / n=k$ into the abovementioned expression, Eq. (92) can be reduced to the following:

$$
D=A k 2^{k-2}\left[\Gamma\left(\frac{k}{2}\right)\right]^{2}[\Gamma(k)]^{-1} a^{k}
$$

Similarly, for the total load in Eq. (32), the following equation is obtained:

$$
P=\frac{E}{1-v^{2}} \frac{A m^{2}}{n(m+n)} 2^{\frac{m-n}{n}}\left[\Gamma\left(\frac{m}{2 n}\right)\right]^{2}\left[\Gamma\left(\frac{m}{n}\right)\right]^{-1} a^{\frac{m+n}{n}}
$$

When $m / n=k$,

$$
P=\frac{E}{1-v^{2}} \frac{A}{2} \frac{k^{2}}{k+1} 2^{k}\left[\Gamma\left(\frac{k}{2}\right)\right]^{2}[\Gamma(k)]^{-1} a^{k+1}
$$

Combining Eq. (93) and Eq.(94) yields

$P=\frac{E}{1-v^{2}} \frac{m}{m+n}\left(\frac{4 n}{A m}\right)^{\frac{n}{m}}\left[\Gamma\left(\frac{m}{2 n}\right)\right]^{\frac{-2 n}{m}}\left[\Gamma\left(\frac{m}{n}\right)\right]^{\frac{m}{n}} D^{\frac{m+n}{m}}$

When $m / n=k$

$P=\frac{E}{1-v^{2}} \frac{1}{k+1} k^{\frac{k-1}{k}} A^{-\frac{1}{k}} 2^{\frac{2}{k}}\left[\Gamma\left(\frac{k}{2}\right)\right]^{-\frac{2}{k}}[\Gamma(k)]^{\frac{1}{k}} D^{\frac{k+1}{k}}$

Notably, Eqs. (93), (95), and (97) agree with the results of Galin (the English language version of the results were edited in Gladwell's book [23]).

\subsection{Application of the general results to six sample punches}

As previously mentioned, the four special known cases of punch profiles $(m / n=0,1,2, k)$ have been reconsidered using the derived formulas in Section 2. Furthermore, some new cases of punch profiles that have not been investigated elsewhere are investigated here according to these general formulas.

To facilitate the comparison with the classical solutions, six examples of punch profiles $(m / n=1 / 4$, $1 / 2,1,4 / 3,2,4)$ are considered, as shown in Fig. 3(a).

To illustrate the differences in the stress states arising from the shape effect, a unified indentation depth ( $D=1$, reduced unit) is set, and the related parameters are calculated and listed in Table 1.

By substituting the related parameters (Table 1) into Eqs. (28), (29), and (32), the normal surface stress, indentation depth, and total load of these six punch profiles can be calculated. They are graphically shown in Figs. 3(b)-3(d), respectively.

Moreover, the distributions of the interior stresses indented by the four punch profiles $(m / n=1,4 / 3,2,4)$ are numerically computed by substituting the parameters (Table 1) into Eqs. (39)-(57). The contour plots of the von Mises stresses can then be illustrated, as shown in Fig. 4.

\section{Conclusions}

The present work explicitly provides the analytical expressions of the stress fields beneath the surface of an isotropic elastic half-space involving axisymmetric punch profile $z=A r^{m / n},(m=0,1,2,3, \ldots ; n=1,2,3, \ldots)$. This is the main contribution of the current research.

The methods adopted in this study for the axisymmetric Boussinesq problem are the Hankel transform and the theory of dual integral equations, which have been pioneered by Sneddon and then developed to solve the related problems. However, its core mathematical procedures have hardly been improved when the distributions of the interior-stress fields were derived. For example, the boundary condition of the $z$-displacement component was 
(a)

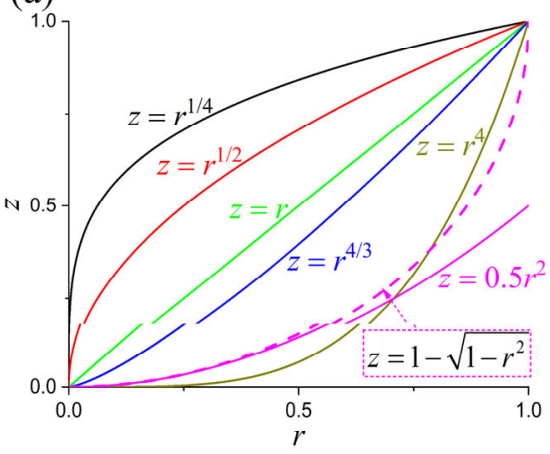

(c)

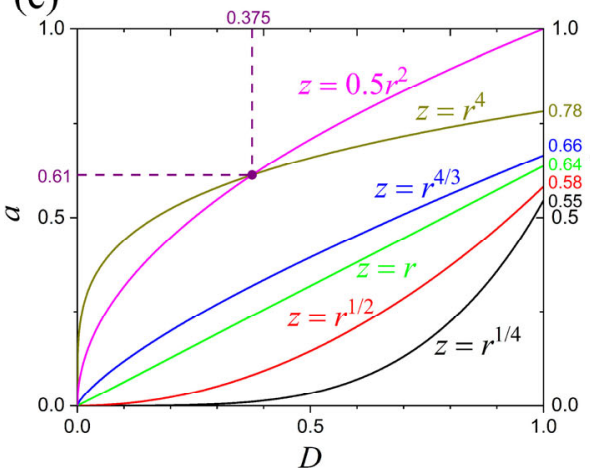

(b)

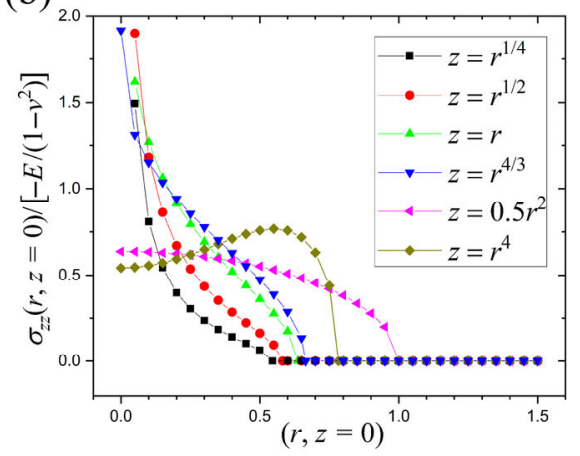

(d)

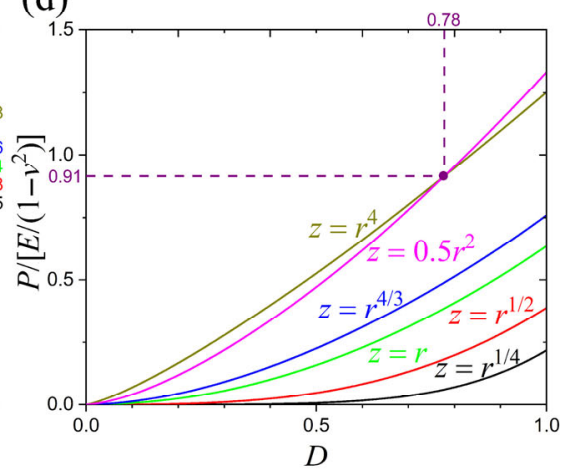

Fig. 3 (a) Generatrix of the six types of axisymmetric punch profiles. Note that the exact expression of spherical punch $z=1-\sqrt{1-r^{2}}$ is replaced by its first-order expansion form, namely, $z=0.5 r^{2}$, in the Hertz contact theory. (b) Distributions of the normal surface stresses produced by unified indentation depth $D=1$ (reduced unit) acting on these six punch profiles where the infinite values at the original point for the cases of $z=r^{1 / 4}, z=r^{1 / 2}$, and $z=r$ are omitted. (c, d) Variations in contact radius $a$, and total load $P$ as indentation depth $D$ varies from zero to one (reduced unit).

Table 1 Parameters of the six punch profiles under unified indentation depth $D=1$ (reduced unit). (The values of contact radius $a$ are rounded off according to Eq. (28)).

\begin{tabular}{ccccccc}
\hline$D=1$ & $z=r^{1 / 4}$ & $z=r^{1 / 2}$ & $z=r$ & $z=r^{4 / 3}$ & $z=0.5 r^{2}$ & $z=r^{4}$ \\
\hline$A$ & 1 & 1 & 1 & 1 & 0.5 & 1 \\
$m / n$ & $1 / 4$ & $1 / 2$ & 1 & $4 / 3$ & 2 & 4 \\
$a$ & 0.55 & 0.58 & 0.64 & 0.66 & 1 & 0.78 \\
$C_{1}$ & $1 / 2 \pi$ & $1 / \pi$ & $2 / \pi$ & $8 / 3 \pi$ & $4 / \pi$ & $8 / \pi$ \\
\hline
\end{tabular}

processed in similar forms of $g(\rho)=A$ by Sneddon, $g(\rho)=A+B \rho$ by Sneddon, and $g(\rho)=A+B \rho^{n}$ by Woirgard for three different punch profiles, which either limited the general discussion of the punch profile or led to extremely implicit and complicated expressions of the interior-stress fields. Instead of using form $g(\rho)=\sum_{n=0}^{\infty} A_{n} \cdot \rho^{n}$, the basic elastic solutions were first re-written in the form $\psi(\zeta)$ (as expressed in Eqs. (2)-(7). Then, the solution of the dual integral equations was transformed into a manageable form (as shown in Eq. (20) by using Eq. (18). The process finally obtained not only these known results but also the explicit and concise formulas of the interior-stress fields for the more general punch profiles (especially for the case of $m / n=4 / 3$, which has not been reported in the literature).

The other main method of the potential theory is also used for this issue, which is based on the Abel integral equation in which the normal pressure should be first deduced. Then, it is considered as a known precondition to derive the distribution of the interiorstress fields.

Irrespective of which of the abovementioned two 

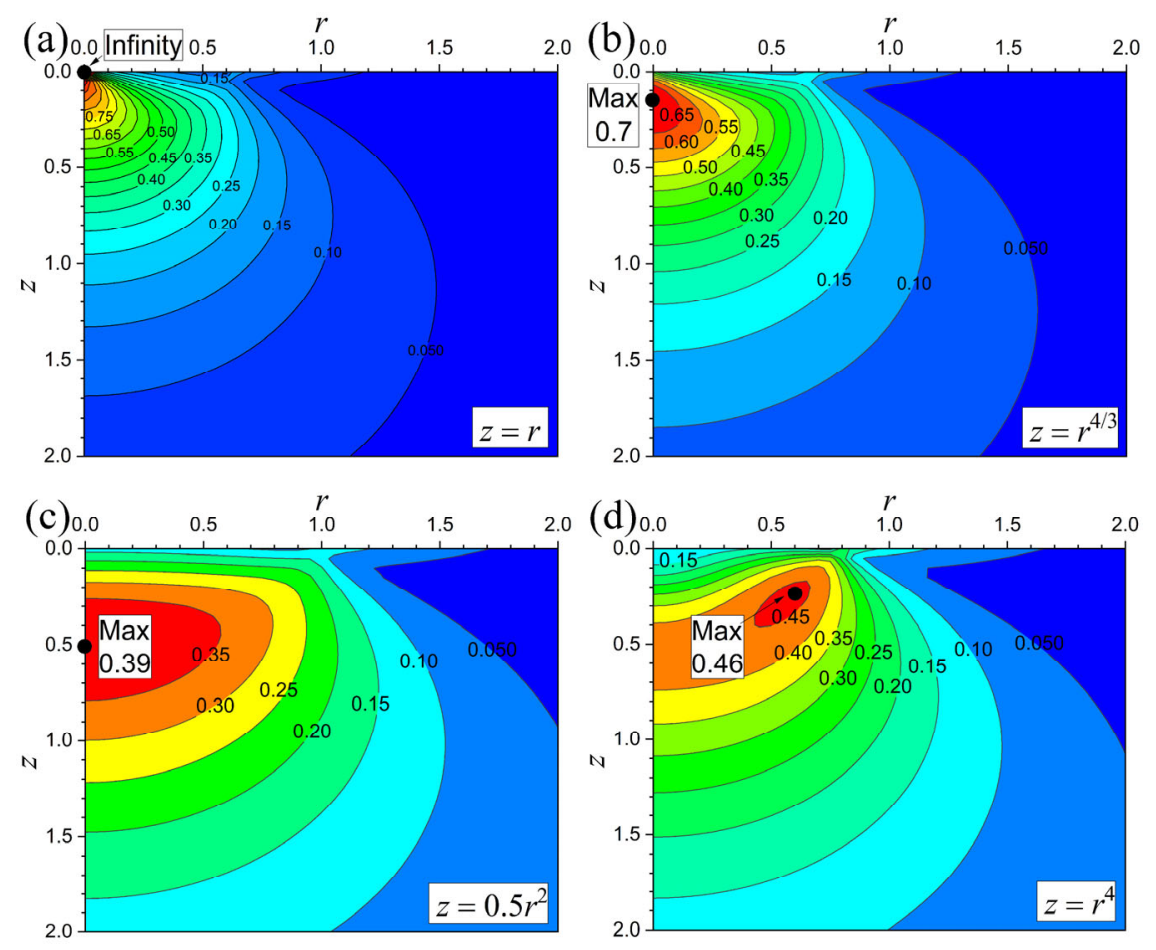

Fig. 4 Von Mises stresses in the elastic isotropic half-space, which are indented by the four punch profiles $(\mathrm{m} / \mathrm{n}=1,4 / 3,2,4)$ when the unified indentation depth $D=1$ (reduced unit) is set.

methods is applied, the direct connection between the basic solutions and function of the punch profile has not been established. This problem is re-solved by the current study. The key point is that the expressions of the stress components need to be rewritten in the form of $\psi(\zeta)$ (as expressed in Eqs. (2)-(7)) and then to simplify the expression of $\psi(\zeta)$ based on the boundary conditions. To our knowledge, this is the first time that such method has been used for this type of problem.

\section{Acknowledgements}

The idea of current work is conceived from Zhanjiang WANG and finished by LongXiang YANG. The author LongXiang YANG would like to appreciate the advanced guidance on the writing from Pro.f Xiqiao FENG in Tsinghua University. The authors would like to acknowledge the partial supports provided by the National Natural Science Foundation of China (Nos. 51575090, 11272083, and 11502049), the Fundamental Research Funds for the Central Universities (Nos. ZYGX2014Z004 and ZYGX2015J084), the China Postdoctoral Science Foundation Grant (No. 2016M590873), and the National Youth Top-Notch Talent Support Program.

Open Access This article is licensed under a Creative Commons Attribution 4.0 International License, which permits use, sharing, adaptation, distribution and reproduction in any medium or format, as long as you give appropriate credit to the original author(s) and the source, provide a link to the Creative Commons licence, and indicate if changes were made.

The images or other third party material in this article are included in the article's Creative Commons licence, unless indicated otherwise in a credit line to the material. If material is not included in the article's Creative Commons licence and your intended use is not permitted by statutory regulation or exceeds the permitted use, you will need to obtain permission directly from the copyright holder.

To view a copy of this licence, visit http://creativecommons.org/licenses/by/4.0/.

\section{References}

[1] Sneddon I N. The relation between load and penetration in 
the axisymmetric Boussinesq problem for a punch of arbitrary prole. Int J Eng Sci 3(1): 47-57 (1965)

[2] Boussinesq J. Application des Potentiels a L'etude de L'equilibre et du Mouvement des Solides Elastique. Pari (France): Gauthier Villars, 1885.

[3] Lamb H. On Boussinesq's problem. Proc Lond Math Soc 34(1): 276-284 (1901)

[4] Schubert G. Zur Frage der Druckverteilung unter elastisch gelagerten Tragwerken. Ingenieur-Archiv 13(3): 132-147 (1942)

[5] Selvadurai A P S. On Boussinesq's problem. Int J Eng Sci 39(3): 317-322 (2001)

[6] Hertz H. On the contact of elastic solids. J Reine Angew Math 92: 156-171 (1882)

[7] Johnson K L. Contact Mechanics. Cambridge (UK): Cambridge university press, 1987.

[8] Barber J R. Contact Mechanics. Cham (Switzerland): Springer International Publishing AG, 2018.

[9] Popov V L, Heß M, Willert E. Handbook of Contact Mechanics: Exact Solutions of Axisymmetric Contact Problems. Berlin (Germany): Springer-Verlag GmbH Deutschland, 2019.

[10] Huber M T. On the theory of elastic solid contact. Annln der Physik 14: 153-163 (1904)

[11] Hills D A, Nowell D, Sackeld A. Mechanics of Elastic Contacts. New York (USA): Butterworth-Heinemann, 1993: 25, 203-204.

[12] Love A E H. IX. The stress produced in a semi-infinite solid by pressure on part of the boundary. Phil Trans $R$ Soc London A 228(659-669): 377-420 (1929)

[13] Love A E H. Boussinesq's problem for a rigid cone. $Q$ J Math os-10(1): 161-175 (1939)

[14] Harding J W, Sneddon I N. The elastic stresses produced by the indentation of the plane surface of a semi-infinite elastic solid by a rigid punch. Math Proc Camb Phil Soc 41(1): 16-26 (1945)

[15] Sneddon I N. Boussinesq's problem for a flat-ended cylinder. Math Proc Camb Phil Soc 42(1): 29-39 (1946)

[16] Sneddon I N. Boussinesq's problem for a rigid cone. Math Proc Camb Phil Soc 44 (4): 492-507 (1948)

[17] Papkovich P. Solution generale des equations diffentielles fondamentales de-lasticite exprimee par trois fonctions harmoniques. CR Acad Sci Paris 195(3): 513-515 (1932)

[18] Papkovich P. Expression for the general integral of the principal equations of the theory of elasticity by harmonic functions, Akad Nauk SSSR ser fiz-met 10(14): 1425-1435 (1932)

[19] Neuber H. Ein neuer Ansatz zur Lösung räumlicher Probleme der Elastizitätstheorie. Der Hohlkegel unter Einzellast als Beispiel. Z Angew Math Mech 14(4): 203-212 (1934)

[20] Green A, Zerna W. Theoretical Elasticity. Oxford (UK): Oxford University Press, 1968.
[21] Sneddon I N. Fourier Transforms. New York (USA): McGraw-Hill, 1951: 453-455, 459.

[22] Muskhelishvili N I. Some Basic Problems of the Mathematical Theory of Elasticity. Dordrecht (the Netherlands): Springer Science, 1977.

[23] Gladwell G M L. Contact Problems (The Legacy of Galin L A). Dordrecht (the Netherlands): Springer Science, 2008: 105-111, 266, 267.

[24] Lur'e A I. Three-Dimensional Problems of the Theory of Elasticity. New York (USA): Interscience Publishers, 1964.

[25] Sneddon I N. Mixed Boundary Value Problems in Potential Theory. Amsterdam (the Netherlands): North-Holland Publishing Company, 1966.

[26] Gladwell G M L. Contact problems in the classical theory of elasticity. Alphen aan den Rijn (the Netherlands): Springer Science \& Business Media B.V., 1980.

[27] Barber J R. The solution of elasticity problems for the halfspace by the method of Green and Collins. Appl Sci Res 40(2): 135-157 (1983)

[28] Payne L E. On axially symmetric punch, crack and torsion problems. J Soc Ind Appl Math 1(1): 53-71 (1953)

[29] Segedin C M. The relation between load and penetration for a spherical punch. Mathematika 4(2): 156-161 (1957)

[30] Deich E G. On an axially symmetrical contact problem for a non-plane die circular in plan. J Appl Math Mech 26(5): 1404-1409 (1962)

[31] Deresiewicz H. Bodies in contact with applications to granular media. In R.d.mindlin \& Applied Mechanics. New York (USA): Pergamon Press, Inc., 1974: 105-147.

[32] Woirgard J, Audurier V, Tromas C. Elastic Stress Field beneath anarbitrary axisymmetric punch. Philos Mag 88(10): 1511-1523 (2008)

[33] Hill R, Storakers B. Elasticity: Mathematical Methods and Applications (The Ian N. Sneddon 70th Birthday Volume). Eason G, Ogden R W, Ed. Chichester (UK): Ellis Horwood Publishers, 1990: 199-210.

[34] Geike T, Popov V L. Mapping of three-dimensional contact problems into one dimension. Phys Rev E 76(3): 036710 (2007)

[35] Popov V L, Heß M. Method of Dimensionality Reduction in Contact Mechanics and Friction. Berlin (Germany): SpringerVerlag Berlin Heidelberg, 2015.

[36] Willert E, Forsbach F, Popov V L. Stress tensor and gradient of hydrostatic pressure in the contact plane of axisymmetric bodies under normal and tangential loading. $Z$ Angew Math Mech 100(7): 1-13 (2020)

[37] Sneddon I N. The elementary solution of dual integral equations. Glasgow Math J 4(3): 108-110 (1960)

[38] Gradshteyn I S, Ryzhik I M. Table of Integral, Series, and Products. Burlington (USA): Elsevier Inc., 2007.

[39] Bower A F. Applied Mechanics of Folids. Boca Raton (USA): CRC Press, 2010: 309-310. 


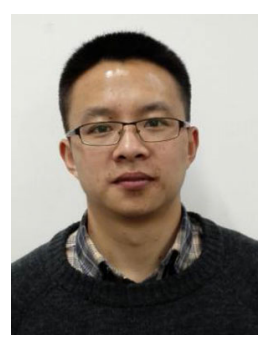

Longxiang YANG. He is a Ph.D. candidate in University of Electronic Science and Technology of China,

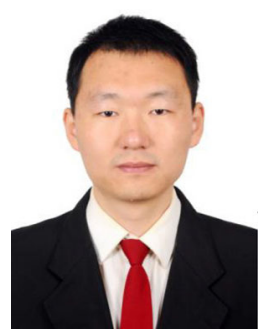

Zhanjiang WANG. He received his Ph.D. degree in mechanical engineering from Tsinghua University,

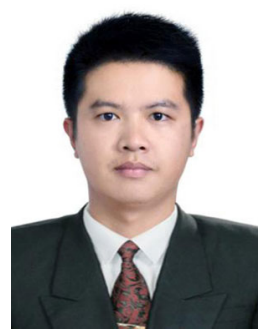

Weiji LIU. He received his Ph.D. degree in mechanical engineering from Southwest Petroleum University, China, in 2017. He is an associate

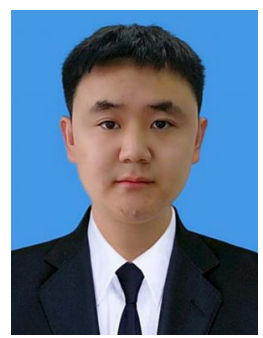

Guocheng ZHANG. He received his B.S. (2015) degree and Ph.D. degree (2020) both from University of Electronic Science and Technology

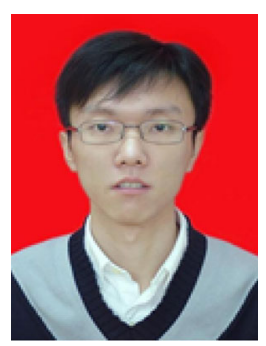

Bei PENG. He received his Ph.D. degree in mechanical engineering from Northwestern University, USA, in 2008. He is a professor at and he also is a lecture at the Geely University of China. His main research areas focus on contact mechanics.

China, in 2010. He is a professor at Southwest Jiaotong University. His scientific interests include computational contact mechanics and damage.

professor at Southwest Petroleum University. His research is mainly on cutter-rock interaction mechanics in oil \& gas drilling engineering.

of China, Chengdu, China. He is a lecturer at Anyang Institute of Technology, China. His research interests focus on the biomechanics and rehabilitative robot.
University of Electronic Science and Technology of China. His interests include micro-nano mechanicas and biology nanotechnology. 


\section{Appendix}

\section{Derivations of the displacement and stress com- ponents in the form $\psi(\zeta)$}

The $z$-displacement component is derived as an example, and the other five expressions can be easily obtained using similar procedures. The expressions are presented in Ref. [21] (as shown in P454-(17), P457-(27)):

$$
\begin{gathered}
u_{z}(r, z)=\int_{0}^{\infty} \xi\left[\frac{\mathrm{d}^{2} G(\xi, z)}{\mathrm{d} z^{2}}-\frac{\lambda+2 \mu}{\mu} \xi^{2} G(\xi, z)\right] J_{0}(\xi r) \mathrm{d} \xi \\
G(\xi, z)=\frac{B}{\xi}\left(\frac{\lambda}{\lambda+\mu}+\xi z\right) \mathrm{e}^{-\xi z}
\end{gathered}
$$

where $-\xi$ should be corrected to $\xi$, and $D$ should be replaced by $B$, which actually is not an arbitrary constant but an undetermined parameter of $\xi$. The second partial derivative of (A2) is expressed as

$$
\frac{\mathrm{d}^{2} G(\xi, z)}{\mathrm{d} z^{2}}=B \xi \mathrm{e}^{-\xi z}\left[-2+\left(\frac{\lambda}{\lambda+\mu}+\xi z\right)\right]
$$

Substituting Eqs. (A2) and (A3) into Eq. (A1) yields the following $z$-displacement component:

$$
u_{z}(r, z)=\int_{0}^{\infty}-B\left[\xi z\left(\frac{\lambda+\mu}{\mu}\right)+\frac{\lambda+2 \mu}{\mu}\right] \xi^{2} \mathrm{e}^{-\xi z} J_{0}(\xi r) \mathrm{d} \xi
$$

By considering relationship $\zeta=\xi a$, Eq. (A4) becomes

$$
\begin{aligned}
& u_{z}(r, z) \\
& =\int_{0}^{\infty} \frac{-B}{a}\left(\frac{\zeta z}{a} \frac{\lambda+\mu}{\mu}+\frac{\lambda+2 \mu}{\mu}\right)\left(\frac{\zeta}{a}\right)^{2} \mathrm{e}^{-\frac{\zeta z}{a}} J_{0}\left(\frac{\zeta r}{a}\right) \mathrm{d} \zeta
\end{aligned}
$$

If we introduce

$$
\psi(\zeta)=-B \frac{\lambda+2 \mu}{\mu} \frac{\zeta^{3}}{a^{3}}
$$

Equation (A5) can be expressed as

$u_{z}(r, z)=\int_{0}^{\infty} \psi(\zeta)\left(\frac{\lambda+\mu}{\lambda+2 \mu} \frac{\zeta z}{a}+1\right) \zeta^{-1} \mathrm{e}^{-\frac{\zeta z}{a}} J_{0}\left(\frac{\zeta r}{a}\right) \mathrm{d} \zeta$
By applying the operator adopted by Sneddon [21], we have

$$
H_{n}[f(\zeta, z) ; \zeta \rightarrow r]=\int_{0}^{\infty} \zeta f(\zeta, z) J_{n}\left(\frac{\zeta}{a} r\right) \mathrm{d} \zeta
$$

Thus, the $z$-displacement component can be finally expressed as

$$
u_{z}(r, z)=H_{0}\left[\psi(\zeta)\left(\frac{\lambda+\mu}{\lambda+2 \mu} \frac{z}{a}+\frac{1}{\zeta}\right) \zeta^{-1} \mathrm{e}^{-\frac{z \zeta}{a}} ; \zeta \rightarrow r\right]
$$

Similarly, by combining Eq. (A2) in the present study with the general expressions, namely, Eqs. (16), (18), (19), (21), and (22) in Chapter 10 of the Sneddon's book [21], i.e.,

$$
\begin{aligned}
& u_{r}(r, z)=\frac{\lambda+\mu}{\mu} \int_{0}^{\infty} \xi^{2} \frac{\mathrm{d} G(\xi, z)}{\mathrm{d} z} J_{1}(\xi r) \mathrm{d} \xi \\
& \sigma_{z z}(r, z)=\int_{0}^{\infty} \xi\left[(\lambda+2 \mu) \frac{\mathrm{d}^{3} G(\xi, z)}{\mathrm{d} z^{3}}\right. \\
& \left.-(3 \lambda+4 \mu) \xi^{2} \frac{\mathrm{d} G(\xi, z)}{\mathrm{d} z}\right] J_{0}(\xi r) \mathrm{d} \xi \\
& \sigma_{\theta \theta}(r, z)=\lambda \int_{0}^{\infty} \xi\left[\frac{\mathrm{d}^{3} G(\xi, z)}{\mathrm{d} z^{3}}-\xi^{2} \frac{\mathrm{d} G(\xi, z)}{\mathrm{d} z}\right] J_{0}(\xi r) \mathrm{d} \xi \\
& +\frac{2(\lambda+\mu)}{r} \int_{0}^{\infty} \xi^{2} \frac{\mathrm{d} G(\xi, z)}{\mathrm{d} z} J_{1}(\xi r) \mathrm{d} \xi \\
& =\int_{0}^{\infty} \xi\left[\lambda \frac{\mathrm{d}^{3} G(\xi, z)}{\mathrm{d} z^{3}}+(\lambda+2 \mu) \xi^{2} \frac{\mathrm{d} G(\xi, z)}{\mathrm{d} z}\right] J_{0}(\xi r) \mathrm{d} \xi \\
& -\frac{2(\lambda+\mu)}{r} \int_{0}^{\infty} \xi^{2} \frac{\mathrm{d} G(\xi, z)}{\mathrm{d} z} J_{1}(\xi r) \mathrm{d} \xi \\
& \tau_{z r}(r, z) \\
& =\int_{0}^{\infty} \xi^{2}\left[\lambda \frac{\mathrm{d}^{2} G(\xi, z)}{\mathrm{d} z^{2}}+(\lambda+2 \mu) \xi^{2} G(\xi, z)\right] J_{1}(\xi r) \mathrm{d} \xi
\end{aligned}
$$

the other five components of Eqs. (3)-(7) can bealogously obtained. 